\title{
Cycle de Marthamea beraudi Navás dans un cours d'eau du Liban (Plecoptera) ${ }^{1}$
}

\section{N.J. Alouf 2}

La rivière Yahfufah est permanente dans sa partie amont. Cing stations situées entre 1100 et $1250 \mathrm{~m}$ d'altitude ont été prospectées. Deux séries de prélèvements, à raison d'un par mois, eurent lieu durant les années 1973-1975 et $1980-1982$.

L'examen des larves de Marihamea beraudi montre que le développement de cet insecte est du type semivoltin $\mathbf{N} 2$. La période de vol s'échelonne entre la seconde moitié du printemps et la mi-été. La température de l'eau varie entre $9^{\circ} \mathrm{C}$ en hiver et $15^{\circ} \mathrm{C}$ en été.

Life cycle of Marthamea beraudi Navás in a Lebanese stream (Plecoptera).

The Perlid Marthamea beraudi Navás occurs in a Lebanese mountain stream. It is a semivoltine N2 type species. The flight-period extends from May to July. The insect seems to prefer the upper part of the stream, where water temperatures vary from $9^{\circ} \mathrm{C}$ in the winter to $15^{\circ} \mathrm{C}$ in the summer.

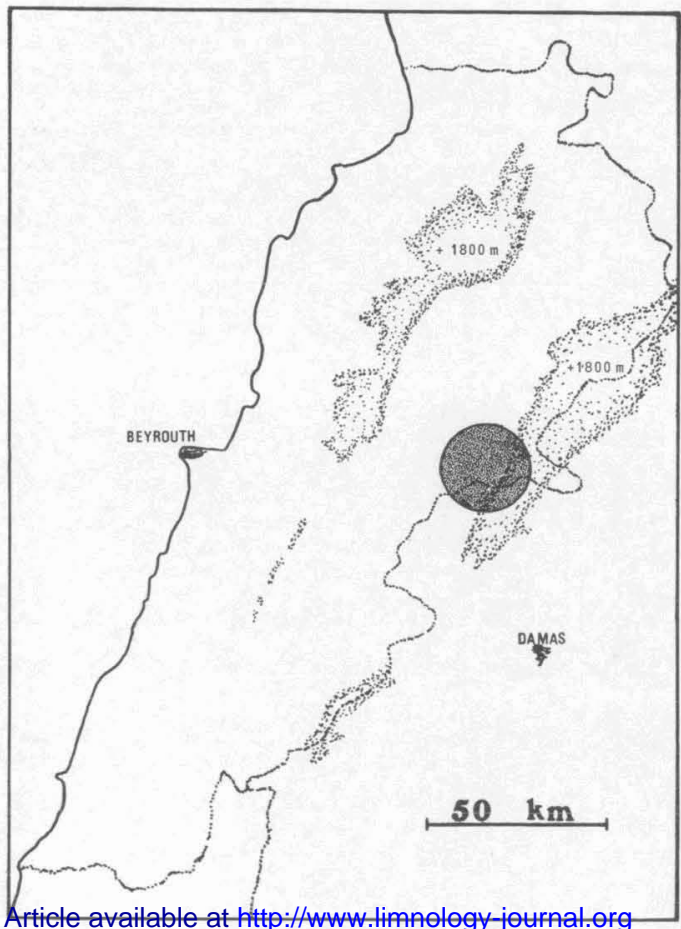

Article available at hitp://www.limnology-journal.org
1. Travail subventionné par le C. N. R. S du Liban.

2. Faculte des Sciences, Section I, Université Libanaise, Hadath-Beyrouth, Liban.

Fig. 1. Carte du Liban. Le cercle délimite la zone prospectée. 


\section{Introduction}

Le genre Marthamea Klapálek a une répartition paléarctique (Illies 1966, Zwick 1973 et 1984). Trois especes $M$. selysii Pictet, $M$. vitripennis Burmeister et $M$. beraudi Navás sont connues, tant pour la forme larvaire que pour la forme adulte (Illies 1955, Zwick 1984). Le cycle d'aucune d'elles n'a été étudié.

Les prospections méthodiques de certains réseaux hydrographiques du territoire de la République Libanaise nous ont permis de récolter un nombre suffisant de larves de $M$. beraudi pour l'étude du cycle de cette espèce.

\section{1. - Le milieu}

Le cours d'eau de Jahfufah ou Yahfoufah (la seule rivière du versant ouest de l'Anti Liban) est pérenne dans sa parti amont au-dessus de $1000 \mathrm{~m}$ d'altitude (Fig. 1). La partie prospectée, de $5 \mathrm{~km}$ environ, est comprise entre 1100 et $1250 \mathrm{~m}$. La largeur du lit

Tableau I. Relevé des températures, en degrés Celsius.

\begin{tabular}{|c|ccccc|}
\hline & \multicolumn{5}{|c|}{ STATIONS } \\
DATES & I & II & III & IV & V \\
\hline 13.10 .80 & 13,8 & 14,1 & 14 & 14 & 13,5 \\
19.11 .80 & 12,5 & 13,1 & 12,5 & 12,3 & 11,5 \\
19.12 .80 & 11 & 12 & 12,1 & 11,5 & 9,6 \\
& & & & & \\
15.01 .81 & 12,2 & 12 & 11,2 & 11 & 10,8 \\
10.02 .81 & 11 & 11,5 & 11 & 10,5 & 9,7 \\
18.03 .81 & 13,3 & 13,2 & 12,9 & 12,3 & 12 \\
29.04 .81 & 13,2 & 13,2 & 13 & & 12,4 \\
21.05 .81 & 13,8 & 13,8 & 13,4 & 13,2 & 12,8 \\
17.06 .81 & 14 & 14 & 13,5 & 13,6 & 13,4 \\
16.07 .81 & 14,5 & 14,5 & 14 & 14 & 14 \\
20.08 .81 & 14,3 & 14,3 & 14,3 & 14 & 14 \\
24.09 .81 & 14,4 & 14,4 & 14,2 & 14 & 13,8 \\
22.10 .81 & 13,8 & 13,8 & 13,8 & 13,1 & 13,1 \\
18.11 .81 & 12,9 & 12,8 & 12,6 & 12,2 & 12,2 \\
16.12 .81 & 12,4 & 12,4 & 12,1 & 12,1 & 11,3 \\
& & & & & \\
14.01 .82 & 11,9 & 11,9 & 11,6 & 11,2 & 11,3 \\
16.02 .82 & 10,7 & 10,6 & 10 & 9,7 & 8,5 \\
16.03 .82 & 13 & 12,9 & 12,5 & 12,4 & 12,2 \\
20.04 .82 & 13,1 & 12,9 & 12,5 & 12,5 & 12 \\
18.05 .82 & 13,8 & 13,7 & 13,1 & 13,5 & 13,5 \\
\hline
\end{tabular}

est de $2.3 \mathrm{~m}$ en amont et de $4.6 \mathrm{~m}$ en aval. La température de l'eau, relevée le long de la partie prospectée, est de $9-13^{\circ} \mathrm{C}$ en hiver et $13.15^{\circ} \mathrm{C}$ en été (Tableau I). Le Nahr (terme arabe pour désigner un " cours d'eau permanent $n$ ) Yahfufah traverse une vallée profonde et dénudée ; la zone verte se limite à une bande étroite dépassant rarement une ou quelques dizaines de métres de part et d'autre du cours d'eau.

\section{2. - Méthodes}

Cinq stations ont été régulièrement prospectées le long de la rivière. Une première série de prélèvements eut lieu chaque troisième semaine du mois, entre juin 1973 et aoút 1975 ; l'échantillon était récolté grâce à un filet de Surber de $0,1 \mathrm{~m}^{2}$ de surface et ayant un vide de maille de $0,3 \mathrm{~mm}$ environ. Une seconde série eut lieu vers le milieu du mois, ent re septembre 1980 et mai 1982 ; l'échantillon était constitué par le total de $3-4$ coups fait avec le même filet Surber. Le but initial était de récolter un nombre suffisant de Gammares.

La taille des larves s'échelonne entre 2 et $25 \mathrm{~mm}$, celle des exuvies imaginales entre 15,5 et $24 \mathrm{~mm}$. Toutefois, pour l'étude du cycle, seule la largeur de la capsule céphalique servit de repère. Les larves sont mesurées avec un oculaire micrométrique et séparées en classe de $0,25 \mathrm{~mm}$. L'emplacement des lieux de récolte a été légèrement déplacé vers l'amont durant la seconde période, en 1980-1982. La répartition des récoltes, dans les cinq stations, est consignée dans le tableau II. En considérant qu'un échantillon de la seconde série équivaut, quantitativement, à trois de la première série, la densité de $M$. beraudi s'établit à 45 individus au $\mathrm{m}^{2}$ dans la zone optimale, en amont. Elle est de $16 \pm 3$ dans les zones contiguës et de $10 \pm 2$ plus en aval. Elle tombe à 1 ou zéro à la limite aval de la zone prospectée (Fig. 2)

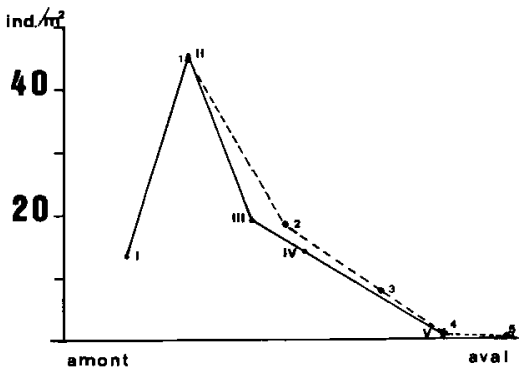

Fig. 2. Densité des Marthamea au $\mathrm{m}^{2}$. Les chiffres arabes indiquent les récoltes de 1973-1975, les chiffres romains les récoltes de 1980-1982. 


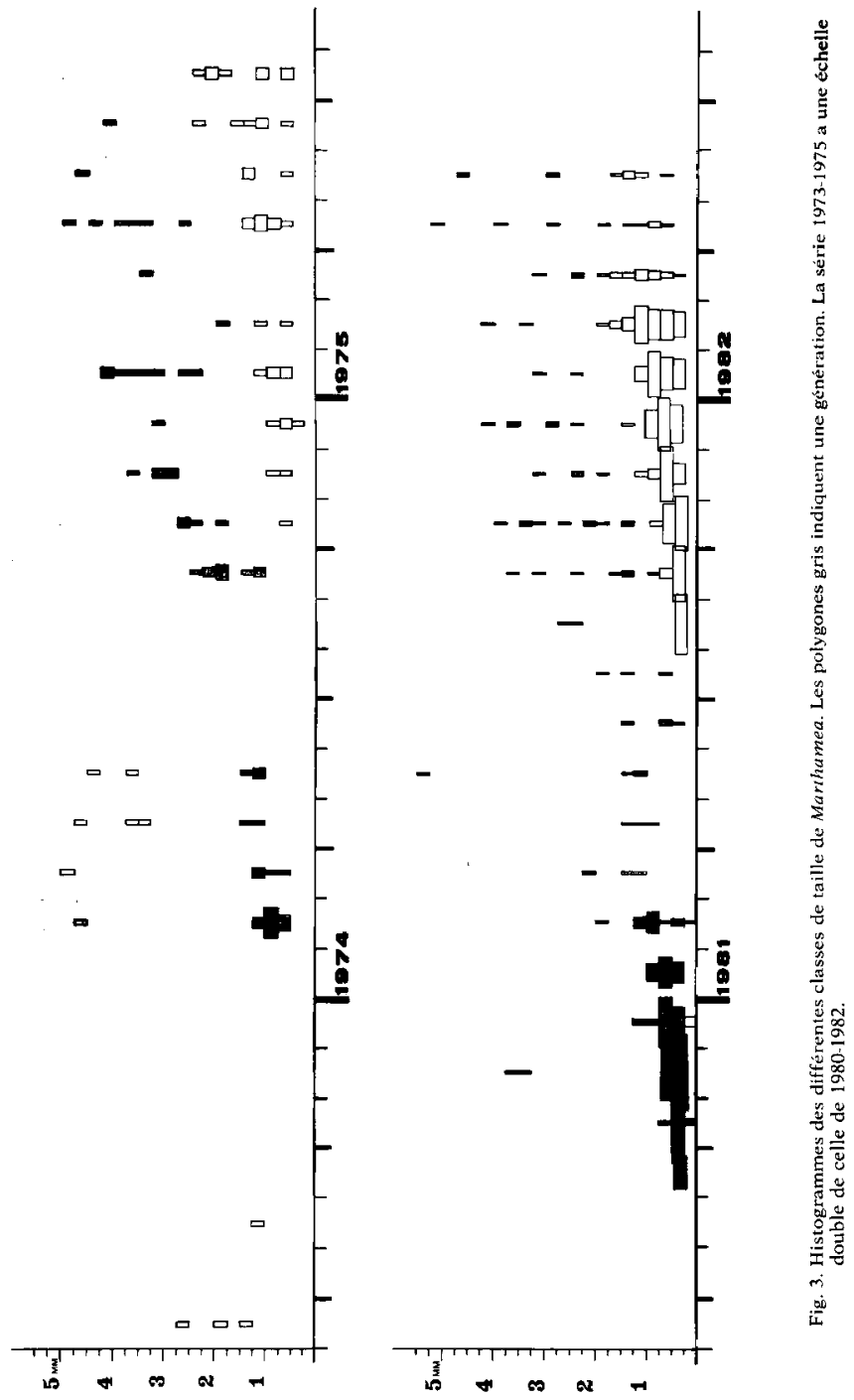




\section{3. - Cycle}

La croissance mensuelle des larves de Marthamea beraudi montre que cette espece est du type semivoltin N2 (Hynes 1970). La période de vol s'échelonne entre la seconde moitié du printemps et la mi-été, (Fig. 3). L'absence de petites larves et de larvules doit être imputée à l'usage d'un filet inadéquat (Neveu 1976) ou bien à des techniques de récolte déficientes, surtout durant la première série. Les premiers imagos de l'année ont été récoltés le 25-5-75, le 23-5-78, le 17-5-79, le 21-5-81 et le 18-5-82 (exuvie). Les derniers l'ont été le 29-6-81. La taille des adultes est de 14-18 mm pour les mâles et de 19-24 mm pour les femelles. La longueur de l'aile antérieure est de $14-18 \mathrm{~mm}$ pour les mâles et de $20-26 \mathrm{~mm}$ pour les femelles, la moyenne étant de $24 \mathrm{~mm}$ (Fig. 4).
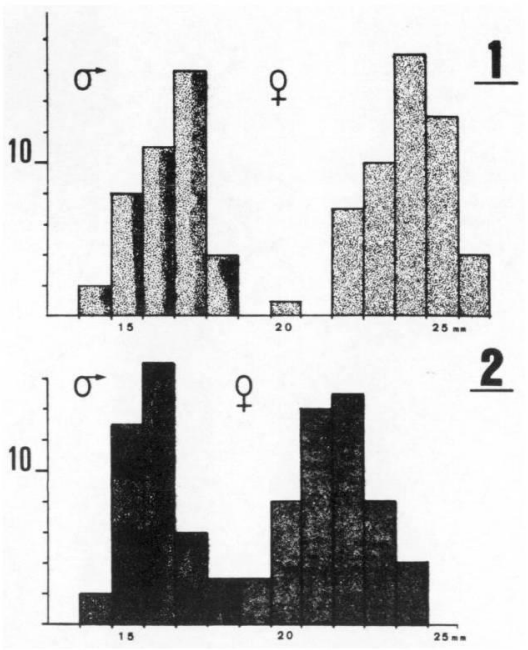

Fig. 4. Histogrammes de la longueur des ailes antérieures (1) et de la taille (2), en mm.

\section{4. - Discussion}

Le cycle de Marthamea ne peut être comparé, par manque de littérature, qu'avec les Perlidés des pays nordiques dont le cycle de vie est du type N3, aussi bien dans les Iles Britanniques (Hynes 1961) qu'en Scandinavie (Ulfstrand 1968). Le raccourcissement du cycle de $M$. beraudi au Liban semble dû à la température relativement clémente de l'eau en hiver. Ainsi, il n'y aurait pas un arrêt ou un ralentissement de croissance et deux années sont suffisantes pour compléter le cycle. Chaque récolte est généralement formé de deux cohortes, la plus ågée est subdivisée en deux ensembles, le plus petit correspond aux mâles et l'autre aux femelles. Sa raréfaction, même son absence, dans les échantillons de la première moitié de l'année 1981 peut être imputée à des facteurs humains. La vallée de Yahfufah était devenue, durant cette période, une voie très fréquentée par les contrebandiers entre le Liban et la Syrie. Parmi les produits de contrebande transitant par cette voie figurait le gas-oil transporté à dos de mulet, ce qui n'a pas manqué de se répercuter sur la zoocénose. La préférence que manifeste cette espèce pour la partie amont du cours d'eau, traduite par une densité croissante d'aval en amont (Tableau II), serait due soit à un réel besoin écologique pour les eaux de l'amont, soit à ce que la présence humaine, plus sensible en aval, constitue une sorte de barrière.

L'absence de la seconde cohorte en 1981 plaide en faveur de l'importance de l'influence humaine. Toutefois, l'absence totale de Marthamea et le changement de la composition de la biocénose à l'aval (travail en préparation) appuie l'hypothèse du préférendum écologique. 
Tableau II. Répartition spatiale et temporelle des larves des Marthamea.

\begin{tabular}{|c|c|c|c|c|c|c|c|c|c|c|c|c|c|c|c|c|c|c|c|c|c|c|c|c|c|c|}
\hline & & $\mathbf{J}$ & J & A $s$ & s 0 & N & D & $\mathbf{J}$ & $F$ & $M$ & $A$ & $M$ & $\mathbf{J}$ & $\mathrm{J}$ & A & $\mathbf{s}$ & 0 & $\mathbf{N}$ & D & J & F & $M A$ & $m$ & J J & Total & Noyem \\
\hline Eation & 1 & - & - & - & $-\quad-$ & - & - & - & - & - & - & - & - & - & - & 7 & 5 & 6 & 4 & 12 & 1 & 16 & 12 & 24 & 69 & 4,3 \\
\hline Sestion & 2 & - & - & - & $-\quad-$ & - & - & - & 12 & $s$ & 5 & 2 & & & & 2 & & & 1 & & & & & 34 & 34 & 1,9 \\
\hline Station & 3 & 1 & & 1 & & & & - & & & & 3 & & & & & & 1 & & 2 & 2 & 8 & 22 & 2 & 22 & 0,9 \\
\hline station & 4 & 2 & & & & & & & & & & & & & & & & & & & & & 1 & & 3 & 0.1 \\
\hline station & 5 & - & - & - & $-\quad-$ & - & - & - & & & & & & & & & & & & & & & & & 0 & 0 \\
\hline & & 3 & & 1 & & & & & 12 & 5 & 5 & 5 & & & & 9 & 5 & 7 & 5 & 14 & 3 & 114 & 447 & 78 & 108 & \\
\hline Station 1 & & - & - & $=-$ & -7 & 9 & 1 & 4 & 1 & & & & 1 & & & 6 & 13 & 5 & 11 & 8 & 1 & 4 & & $-\quad-$ & 71 & 3.6 \\
\hline Station II & & - & - & -10 & 109 & 12 & 13 & 10 & 10 & 1 & & 3 & 3 & 3 & 2 & 27 & 23 & 28 & 35 & 30 & 42 & 84 & $45-$ & $-\quad-$ & 278 & 13,2 \\
\hline 8tation III & & - & - & $-\quad-$ & -21 & 17 & 16 & 12 & 5 & 2 & 2 & & & & 7 & 2 & 1 & 7 & 5 & 11 & 3 & 63 & 32 & $-\quad-$ & 116 & 5,8 \\
\hline station IV & & - & - & -3 & 31 & 18 & 12 & 1 & 2 & & 1 & & & & 16 & & 11 & & 3 & 2 & 14 & & $24=$ & - & 90 & 4,3 \\
\hline Scation V & & - & - & - & & & & & & & & & & & 1 & & & & & & & & & - & 1 & 0,0 \\
\hline
\end{tabular}

Travaux cites

Hynes (H.B.N.). 1961. - The invertebrate fauna of a Welsh mountain stream. Arch. Hydrobiol., $57: 344-388$.

Hynes (H.B.N.). 1970. - The Ecology of muning waters. Liverpool University Press, Liverpool. 555 p

Illies (J.). 1955. - Die Tiemelt Deutschiands. 43 Teil. Steinfliegen oder Plecoptera. Gustave Fischer Verlag, Jena. 150 p.

Illies (J.) 1966. - Katalog der rezenten Plecoptera. Das Tierreich 82: xxx +632 p. W. de Gruyter, Berlin

Neveu (F.). 1976. - Ecologie des larves d'Athericidae (Diptera Brachycera) dans un ruisseau des Pyrénées Atlantiques. I.
Structure et dynamique des populations. Ann. Hydrobiol. $7: 73-90$.

Ulfstrand (S.). 1968. - Life cycles of benthic insects in Lapland streams (Ephemeroptera, Plecoptera, Trichoptera, Diptera Simuliidae). Oikos, $19: 167-190$.

Zwick (P.). 1973. - Insecta : Plecoptera. Phylogenetisches System wnd Katalog. Das Tierreich, $94:$ xxxii + 465 p. W. de Gruyter, Berlin. New-York.

Zwick (P.). 1984. - Manhamea beraudi (Navás) and its European congeners (Plecoptera : Perlidac). Annls Limnol. 20: 129.139 